\title{
Schelling y Fichte: algunas afinidades en el plano filosófico-jurídico
}

Mariano Gaudio

\section{OpenEdition}

Journals

Edición electrónica

URL: https://journals.openedition.org/ref/397

DOI: 10.4000/ref.397

ISSN: 2258-014X

Editor

EuroPhilosophie Editions

Referencia electrónica

Mariano Gaudio, «Schelling y Fichte: algunas afinidades en el plano filosófico-jurídico», Revista de Estud(i)os sobre Fichte [En línea], 3 | 2011, Publicado el 01 février 2011, consultado el 28 juin 2022 URL: http://journals.openedition.org/ref/397 ; DOI: https://doi.org/10.4000/ref.397

Este documento fue generado automáticamente el 29 septiembre 2020

(c) EuroPhilosophie 


\title{
Schelling y Fichte: algunas afinidades en el plano filosófico- jurídico
}

\author{
Mariano Gaudio
}

1.

1 La relación entre el primer Schelling, cautivado por la Doctrina de la Ciencia, y las sucesivas elaboraciones de Fichte, se encuentra signada por la complejidad. En las últimas décadas los especialistas de uno y de otro filósofo han acentuado las diferencias, colocando a Schelling como un intérprete dogmático que absolutiza el principio fundamental de Fichte, o situando a éste en el ámbito de un eticismo subjetivo despojado de naturaleza. Así las divergencias se multiplican, y ni Schelling habría comprendido correctamente a Fichte, ni Fichte habría comprendido correctamente a Schelling. Sin ánimo de minimizar los puntos de ruptura, sin embargo resulta posible encontrar continuidades significativas, no sólo en cuanto al uso de ciertos términos, sino también y principalmente en cuanto a que comulgan en determinadas perspectivas conceptuales. Aunque no podamos resolver aquí la pregunta sobre si la discordancia se reduce simplemente a matices o alcanza cuestiones de fondo, sí podremos aportar algunos lineamientos en la aproximación al problema.

2 En el presente trabajo tomaremos como ilustración de un núcleo de coincidencias básicas algunos conceptos vertidos por Schelling en la Nueva deducción del derecho natural (1795), los cuales remiten tanto a aspectos nodales de la Doctrina de la Ciencia de Fichte, como a incursiones específicas del ámbito jurídico desplegadas en el Fundamento del derecho natural (1796) ${ }^{1}$. Previamente analizaremos de modo sucinto las referencias mutuas y los elementos que conciernen a la comprensión filosófica.

3 Curiosamente, mientras Schelling se encamina hacia la filosofía de la naturaleza señalado por los especialistas como otro hito de la ruptura entre ambos-, Fichte en su Segunda introducción a la Doctrina de la Ciencia consigna cuatro notas al pie de página 
donde sugiere su aprobación a Del Yo y a Ideas para una filosofía de la naturaleza. En la primera nota, en el contexto de la defensa de la intuición intelectual y de la unidad entre teoría y práctica, Fichte critica al pasar una reseña contra $D e l Y^{2}$. En la segunda nota, quizás la más relevante, exceptúa a Schelling del abanico de seguidores de Kant que, bajo el rótulo de intérpretes autorizados, entresacan fragmentos de su exposición, enarbolan la letra y sacrifican el espíritu de conjunto ${ }^{3}$. En ese afán repetitivo -prosigue Fichte- casi todos los kantianos acuerdan con Reinhold en que para Kant el fundamento de la experiencia se halla fuera del Yo; para Fichte este paso sintetiza la estrechez empirista y el retorno del dogmatismo. De ahí que más abajo ${ }^{4}$ distinga entre el inventor (Erfinder) de un sistema filosófico, y quien lo comenta (Erklärer) o continúa (Nachfolger), es decir, el intérprete (Ausleger) que reproduce los fragmentos creyendo que de esa manera desembocará en el todo. En efecto, Fichte aparta a Schelling de ese abanico, precisamente porque -como veremos luego- lo consideraría un coetáneo del movimiento hermenéutico. En la tercera nota, tras ofrecer una pincelada sobre los más destacados pensadores modernos (Spinoza, Kant, Leibniz), Fichte remite elogiosamente a Ideas para una filosofía de la naturaleza ${ }^{5}$. Y en la cuarta nota vuelve a defender a Schelling de críticas que pueden incluso rayar en la calumnia ${ }^{6}$.

4 En los Tratados para el esclarecimiento del idealismo de la Doctrina de la ciencia, una compilación de textos independientes publicados en sucesivos números del Philosophisches Journal bajo el título Panorama general de la literatura filosófica más reciente, Schelling se ocupa entre otros temas de la recepción y la comprensión del kantismo, y subraya el mérito de Fichte de haber elevado la filosofía práctica a la cima de toda la filosofía. Además, lo desliga de la visión común y prosaica del idealismo despojado de realidad efectiva, y se sitúa él mismo en la senda de la Doctrina de la Ciencia ${ }^{7}$. Asimismo, en el "Prólogo" de su gran Sistema del idealismo trascendental, Schelling confiesa: "no puede aparecer aquí nada que no haya sido dicho hace tiempo o en los escritos del creador (Erfinders) de la Doctrina de la Ciencia o en los del autor"'.

5 La auténtica comprensión filosófica consiste en capturar el espíritu de la obra, en atravesar la cáscara fenoménica para reconstruir el sentido de la totalidad de la producción, poniendo al creador en concordancia consigo mismo. Esta solicitud compartida por ambos9- de juzgar un sistema en su conjunto se tensiona con la exigencia simultánea de un lector/oyente activo que autogenere en sí el proceder de la libertad. ¿Cómo evitar, entonces, el malentendido y la tergiversación? Se supone que los límites de la comprensión arbitraria estarían dados por la coherencia interna y el carácter deductivo de las proposiciones. Aun así, la interpretación filosófica descubre elementos ocultos al autor examinado. Fichte ensaya esta perspectiva en Algunas lecciones sobre el destino del sabio: "comprenderemos a Rousseau mejor de lo que él se comprendió a sí mismo, y lo encontraremos en perfecta concordancia consigo mismo y con nosotros"10. Pero pareciera retraerse cuando el desafío reside en entender a Kant: por un lado, Fichte sostiene que los seguidores no han captado la raíz de la propuesta crítica -e incluso la han conducido a posiciones dogmático-empiristas-; por otro, se inscribe en ese proyecto, aunque advierte que su exposición resulta diferente. "Kant es hasta ahora [...] un libro cerrado, y lo que se ha leído de él es precisamente aquello que no se ajusta en él"11; por ende, la Doctrina de la Ciencia “... no es otra que la doctrina kantiana bien entendida"12. Sin embargo, tanto los amigos como los adversarios aducen lo contrario, y ante la duda sobre el veredicto final, Fichte acepta que Kant mismo “... tiene que ser quien seguramente y sin duda mejor se comprende a sí mismo"13. Luego ironiza sobre su incapacidad para entender correctamente a Kant, lo cual significaría, 
en consecuencia, declarar su propia filosofía como una elaboración original, un hallazgo que lo ha conducido a la concordancia consigo mismo. Pero Fichte no queda conforme en esta tesitura, y en lo sucesivo muestra los principales puntos de conexión con el kantismo bien entendido, deslindando las pretensiones de sus seguidores. Lo que no se puede explicar según la letra, se desprende del espíritu, tal como Kant lo advierte en el segundo prólogo a la Crítica de la razón pura, y tal como lo lleva a cabo interpretando a Leibniz; por consiguiente, concluye Fichte, "un escritor filosófico original [...] se explica según el espíritu que realmente está en él, y no según el espíritu que aparentemente debería estar en él"14. Una vez más, el criterio apuntalaría la coherencia: si al rechazar una premisa que se sigue del sistema interpretado se cae en una contradicción, entonces semejante deducción forma parte del espíritu efectivamente existente.

6 Schelling, en cambio, procede con total franqueza y admite que el idealismo parte de Kant y, al mismo tiempo, va más allá de Kant:

“... no puedo esperar [un examen dirigido a los principios] de aquellos lectores [...] que presuponen que después de Kant ninguna nueva investigación de los principios sea posible y que los principios supremos de su filosofía fueron establecidos por él mismo". Y agrega más abajo: “... contra tales seguidores (Anhänger) de Kant [...] se puede notar que quizás hayan captado la letra, pero no el espíritu" ${ }^{15}$.

La letra, ajustada a la exposición del sistema, se convierte en un impedimento que sustrae el fondo de la cuestión; por ende, confiesa Schelling:

"[...] considero necesario recordar que nunca he tenido en mente transcribir de nuevo lo que Kant había escrito (para eso bastan las manos), ni tampoco saber lo que Kant realmente quido con su filosofía, sino sólo aquello que según mi entender debería haber querido, si su filosofía ha de ser coherente consigo misma"16.

Encontramos aquí una unidad de medida similar a la de Fichte: la conexión de las piezas en la totalidad, la captación de lo que se expresa o manifiesta materialmente, el sentido de lo que se quiere decir, incluso a pesar del autor, constituyen las exploraciones hermenéuticas que el espíritu realiza sobre sí mismo, no en cuanto éste o aquél filósofo empírico, sino en cuanto autoconcordancia de la filosofía o de la verdad consigo misma.

7 Esta lección -muy escueta y simplificada- acerca de cómo comprender filosóficamente a un creador original representa una guía para instalarse en la intrincada relación entre Fichte y Schelling.

\section{2.}

8 Con Kant la filosofía ha iniciado el camino revolucionario de asentar en el sujeto el conocimiento de los objetos, pero se necesita una "segunda revolución" concerniente a los principios, que desprenda al ser humano de las ataduras del mundo objetivo y abra las puertas del querer (Wollen) y del actuar (Handeln) en unidad con el conocimiento. En efecto, el paso que Kant no dio consiste en unificar teoría y práctica, otorgándole a la segunda la potestad que le corresponde. Según Schelling, aunque la época tal vez no esté lo suficientemente madura, es el momento de una nueva filosofía:

“...una filosofía que establece como su primer principio la afirmación de que el ser del hombre sólo consiste en la libertad absoluta, que el hombre no sea ninguna cosa (Ding), ningún objeto (Sache), y que su ser propio no sea ningún objeto (Objekt) en general"17.

Lejos de la cosificación, Schelling aspira, al igual que Fichte, a que el ser del hombre se defina, no por la relación con los objetos, sino a partir de la voluntad y la libertad, el 
autohacerse. En este sentido, la razón teórica no alcanza a captar y expresar lo incondicionado ${ }^{18}$, el "ser último" o "absoluto", "lo que está como fundamento de todo lo que existe", "lo que se revela en cada existencia", lo "idéntico conmigo mismo", según la terminología de la Nueva deducción ${ }^{19}$.

9 Ahora bien, la apelación al "ser" podría dar lugar a confusión, porque Fichte rechaza explícitamente la versión de un primer principio concebido como cosa, como ser fijo, inmutable, trascendente, etc.; es decir, rechaza toda instancia que convierta en heterónoma a la libertad ${ }^{20}$. También Schelling descarta esta posibilidad en cuanto excluye al No-Yo como primer principio y señala el dogmatismo incoherente de Spinoza $^{21}$. Por lo tanto, no se trata del ser que operaría de modo trascendente y anulando la autonomía del Yo, sino del que se constituye -en palabras de Fichte- desde la propia actividad autogenerada: "El Yo se pone a sí mismo, y es gracias a este simple poner por sí mismo; y al revés: el Yo es, y pone su ser gracias a su simple ser" 22; sujeto y objeto coinciden en la Tathandlung, la expresión que condensa al actuante y lo actuado, el productor y el producto, la actividad y el resultado. El Yo consiste en esa misma actividad; allí reside su ser, su esencia dinámica y absoluta en cuanto desligada de todo condicionante y en cuanto se activa desde sí misma.

10 En otras palabras, Schelling transforma la exhortación fichteana de pensarse a sí mismo para descubrir el propio actuar interno y remontarlo a sus fundamentos ${ }^{23}$ en un llamado a ser: "¡Sé!, en el sentido supremo de la palabra [...]; esfuérzate por devenir un ser en sí" ${ }^{24}$, lo cual significa que ninguna fuerza de tendencia contraria pueda cambiar tu situación o limitar tu libertad, y prosigue: "esfuérzate por devenir un ser en sí, por ser absolutamente libre" 25 , lo cual significa que la libertad o autonomía someta a su gobierno toda fuerza extrínseca, todo límite dado. Este esfuerzo incondicionado resulta "dependiente sólo de sí mismo y no determinable por ninguna ley extraña" ${ }^{26}$. Todo lo opuesto se convierte en objeto, en algo determinado por el esfuerzo, mientras que lo determinable refiere a la propia capacidad de autodeterminarse. El Yo se establece como "señor de la Naturaleza" y la autonomía reside en el sí-mismo (Selbst) invariable, que subordina y subsume para sí cualquier finalidad ajena que, en caso de sobreponerse a ella, la tornaría heterónoma y, por ende, en sí contradictoria ${ }^{27}$.

11 Queda claro, no obstante, que la primacía de la autoactividad (Selbstthätigkeit) ${ }^{28}$ no soslaya las limitaciones, no destruye el mundo objetivo, o de otra manera, no disuelve el realismo implícito en el idealismo; antes bien, significa que tales huellas de la heteronomía se dan junto con la realización práctica de la libertad. Las limitaciones no eliminan la autonomía del sujeto, sino que se presentan como momentos de la concreción o aplicación de esa libertad que, de no lidiar y sortear los límites, permanecería en la abstracción absoluta. En este sentido, afirma Schelling:

"la libertad en general sólo puede anunciarse mediante la autonomía originaria. Por lo tanto, esta causalidad física, aunque heterónoma según el objeto [...], tiene que ser autónoma según su principio [...]. Ella tiene que reunir en sí autonomía y heteronomía" 29 .

La causalidad física es una revelación de la libertad en el mundo de los fenómenos, la vida misma; es una manifestación que se extiende hasta donde lo permita la dialéctica entre la fuerza (Macht) del sujeto y la resistencia (Widerstand) de lo que se le opone ${ }^{30}$.

12 Este énfasis en la libertad del Yo, en el esfuerzo por realizarse, en considerar todo lo contrapuesto (la Naturaleza o No-Yo) en referencia al Yo, y en caracterizar el proceso de realización práctica como pasaje de lo determinable a lo determinado, son tópicos 
medulares de la filosofía de Fichte ${ }^{31}$. En el caso específico del Fundamento del derecho natural, Fichte comienza recreando el primer principio, la identidad entre el actuante y lo actuado, sujeto y objeto, la acción y el producto, el ser racional que se pone a sí mismo y no admite otro ser/cosa que lo determine. En la autonomía del ponerse consiste, precisamente, el "carácter de la racionalidad", y a ello refiere la palabra Yo o Yoidad (Ichheit) en la medida en que el ser racional abstrae de su propio $\mathrm{Yo}^{32}$. En esta perspectiva, el hecho de que el sujeto se sienta coaccionado a representar un objeto de determinada manera -es decir, tal como lo percibe-, y a otorgarle realidad, no significa más que un actuar necesario fundado, en última instancia, en el actuar libre del Yo. El objeto, entonces, no constituye una realidad independiente, sino lo dado, lo que se presenta sin la intervención del sujeto; pero, en cuanto se lo conceptualiza y comprende, se le adscribe a ese objeto un actuar correspondiente, y aunque el proceder parezca necesario, responde a los parámetros de la libertad. Que el sujeto se sienta coaccionado a representar el objeto de determinada manera, porque así se le aparece, constituye también un modo libre de actuar ${ }^{33}$.

13 En los primeros parágrafos Fichte se encarga de desarrollar y aclarar la centralidad de la autonomía y del querer del Yo. Ponerse a sí mismo equivale a atribuirse una causalidad $u$ operatividad efectiva y libre, lo cual a su vez se contrapone a la actividad en la intuición del mundo -el conocimiento de los objetos u actividad objetiva-. Ahora bien, no se trata de dos actividades absolutamente diferentes, sino de dos caras de una misma actividad: "la intuición [del mundo] es la actividad causal (Wirksamkeit) suprimida, abandonada voluntariamente por el ser racional mismo" ${ }^{34}$. En otras palabras, el conocimiento de los objetos responde a la autodeterminación del sujeto a conocer. La percepción depende del querer, y “[e]l querer es el propio carácter esencial de la razón" ${ }^{35}$. En paralelo con los términos de Schelling, autonomía y heteronomía no sólo se determinan recíprocamente, sino que también se complementan y forman parte del ejercicio de la libertad. Asimismo, Fichte avanza sobre lo que Schelling denominó "vida", en el sentido de que configura la realización de la voluntad en el mundo sensible a través del cuerpo propio, definido como "el ámbito de todas las posibles acciones libres de la persona". Y continúa:

"La persona es libre; esto significa [...] que, únicamente mediante el proyecto de un concepto de fin, deviene sin más causa de un objeto que precisamente corresponde a ese concepto; deviene causa sola y únicamente por su voluntad como tal, pues querer significa proyectar un concepto de fin" ${ }^{36}$.

La concreción de los fines proyectados se efectúa por medio del cuerpo propio, donde el querer y la libertad se manifiestan de manera singular, porque el cuerpo individualiza. El producto de la acción se halla directamente ligado al actuante y expresa su voluntad. En este plano de la corporalidad física, de lo concreto y singular, Fichte sitúa la relación intersubjetiva, la influencia (Einfluss, Einwirkung, § 6) recíproca que se desenvuelve en el trato: "en cada uno de los dos [que entablan una acción recíproca] está presente el concepto de que el otro es un ser libre, y no un ser a tratar como una mera cosa" ${ }^{37}$.

\section{3.}

14 Según el esbozo del apartado anterior, el núcleo de coincidencias básicas entre la Nueva deducción de Schelling y el Fundamento del derecho natural de Fichte se extiende más allá del primer principio y la articulación entre libertad y limitación. Entre otros aspectos, cabe agregar: la separación entre derecho y moral/ética, deducción y análisis del 
principio o concepto de derecho a partir de la razón, la problemática del reconocimiento y modalidades del trato con el otro ${ }^{38}$, articulación entre la voluntad universal y la individual, el estado de naturaleza conflictivo y destructivo, disolución de los derechos originarios y de coacción sin Estado, vacuidad y futilidad del contrato ${ }^{39}$, la necesidad de transformar el poder físico en ley/derecho o poder legítimo, el carácter aporético/escéptico del derecho natural, etc. Ante este abanico de perspectivas, en lo sucesivo nos limitaremos a indagar la relación intersubjetiva y sus consecuencias jurídicas, reconstruyendo la argumentación que conduce a la imposibilidad del derecho natural anclado en la libertad individual.

15 El modo en que Schelling introduce la relación con el otro y el surgimiento de la conflictividad generan, por una parte, una suerte de homologación entre sujeto y objeto y, por otra, la necesidad de regular jurídicamente las relaciones intersubjetivas. Ambas direcciones confluyen en el severo cuestionamiento de la adjudicación de libertad a nivel individual. Analicemos el problema por pasos. El punto de partida, la exhortación al despliegue de la libertad incondicionada e ilimitable, se conjuga con dos tipos de resistencia: la limitación física y la limitación moral. En la primera el sujeto se enfrenta a la superioridad de la naturaleza, en la segunda a la aparición del otro, al rostro de la humanidad. ${ }^{40}$ Ahora bien, lo que determina que el otro sea un ser moral con el cual se entabla un trato recíproco- no es su figura exterior, sino la expresión de su voluntad; pues sucede que cuando la voluntad de otro no se afirma, los demás “... pierden finalmente todo respeto (Achtung) hacia esta especie sumisa" ${ }^{41}$. El trato recíproco excede la connotación empírica y se muestra como un asunto de la práctica; más aún, puesto que las múltiples voluntades se piensan como causalidades ilimitadas, podrá encontrarse una convergencia e identidad en el fin último, en el ideal de la concordancia absoluta, mientras se instala y propaga el conflicto en el esfuerzo concreto:

"la causalidad incondicionada de los seres morales deviene conflictiva (widerstreitend) en el esfuerzo empírico, y comienzo a contraponer mi libertad a la libertad de todos los demás" ${ }^{42}$.

16 Si reparamos en la observación anterior, el simple hecho de que el otro no oponga su voluntad, no se afirme a sí mismo, abre paso al sometimiento y a la gesta de un trato que naturaliza la relación entre un superior y un inferior. Que el trato recíproco se desarrolle en condiciones de igualdad -tal como desea Fichte, por ejemplo- requiere de voluntades que se auto-afirmen. Pero a su vez la auto-afirmación se gesta en contraposición al otro, y la unicidad moral contrasta con la disputa empírica. Por lo tanto, si el desafío de la ética consiste en conciliar la libertad universal con la voluntad individual, el otro no puede ser reducido a la condición de objeto:

"El mandato supremo de toda ética es el siguiente: actúa de modo tal que tu voluntad sea voluntad absoluta [...]; actúa de modo tal que, mediante tu acción [...] ningún ser racional sea puesto como mero objeto, sino como sujeto co-actuante (mithandelndes)" ${ }^{43}$.

Para que esta unificación sea posible tiene que cesar la contraposición de uno y otro, al mismo tiempo que se consagra una voluntad universal que mantenga firme la voluntad individual ${ }^{44}$.

17 Igualmente, la comunidad se establece sólo en el plano ético, mientras que para lo fáctico (el foro externo) se requiere de una "ciencia del derecho", que Schelling deduce por antítesis a la ética ${ }^{45}$, y que delimita lo permitido, lo justo o correcto. El "principio supremo del derecho" apuntala conjuntamente la autoafirmación de la voluntad 
individual y la conformidad con la voluntad universal. Del análisis del principio supremo se desprenden: el derecho a la libertad moral y a la igualdad formal y el derecho sobre los objetos ${ }^{46}$. En los tres casos se presentan situaciones conflictivas: en el primero, que la voluntad universal suprima a la individual; en el segundo, que dos voluntades particulares entren en colisión (entre sí y/o ante la ley); y en el tercero, que la apropiación excluya a todos los otros ${ }^{47}$.

18 En este último punto, y en el subsiguiente (el derecho de coacción), el reconocimiento moral del otro se desvincula del trato recíproco, porque la identidad formal se conjuga con la diferencia material, es decir, con la voluntad propia del otro que se autoafirma y, gracias a ello, "lo reconozco [como] un ser igual a mí". Y continúa Schelling: "si él suprime la forma de la voluntad en sí, entonces deja por eso de ser idéntico conmigo; deviene objeto para mí". Lo mismo vale de manera inversa: "todo ser, en la medida en que suprime la forma de la voluntad en mí, deviene mero objeto" ${ }^{48}$. ¿Quién determina que el otro no afirma su voluntad o anula la mía? El trato recíproco desemboca en un juego de fuerzas que luchan por doblegarse, y si el derecho natural equivale a la afirmación individual de los derechos que se siguen de las leyes de la naturaleza, “... en la disputa (Streit) todo ser es para mí un mero ser natural" ${ }^{49}$. El conflicto se desprende de la simple potestad individual de interpretación de lo jurídico; por ende, lo que aparentaba una resolución justa termina siendo una lucha física donde lo correcto está del lado del más fuerte. Concluye Schelling: "El derecho natural necesariamente se destruye a sí mismo en sus consecuencias (en la medida en que deviene derecho de coacción)" ${ }^{\prime 5}$. En otras palabras, la auto-adjudicación de libertad a nivel pura y exclusivamente individual -esto es, en un contexto de estado de naturaleza, donde la unicidad de la voluntad moral no se halla institucionalizada-, coarta toda posibilidad de relación jurídica. Y el germen de la insuperable conflictividad intersubjetiva radica tanto en la exigencia inicial o esfuerzo hacia lo incondicionado, como en la subsunción del otro a la condición de mero objeto, obstáculo, voluntad débil, etc.

19 Fichte, en cambio, posterga la conflictividad en el trato recíproco y la presenta como una destrucción de las precondiciones de igualdad en las que se desenvuelve la libertad en un comienzo. La estrategia consiste en situar al otro en la posición del sí-mismo:

"el ser racional no se puede poner como un ser tal, con autoconciencia, sin ponerse como individuo, como uno entre muchos seres racionales, a los cuales acepta (annimmt) fuera de sí, así como se acepta a sí mismo"51.

El ponerse a sí mismo no se puede desvincular de un simultáneo poner a los otros en la misma condición, es decir, en cuanto seres racionales que se ponen y ponen a los demás. Lo mismo acontece con la libertad: un sujeto no puede adjudicarse libertad sin al mismo tiempo dividirla y adjudicar una porción correspondiente a cada otro sujeto. Dicho negativamente, si un sujeto no admite seres libres e iguales a él, entonces no se admite a sí mismo, ni libre ni igual. Ahora bien, la libertad no concierne sólo a la proyección de un concepto de fin para una actividad posible, sino también al objeto que se sigue de esa actividad causal. La limitación recíproca involucra aquello en lo cual la libertad se plasma, el mundo sensible. Esto no significa que los límites provengan de las cosas, ni de algo que esté fuera de la libertad; antes bien “... todos tendrían que ponerse estos límites mediante la libertad misma", haciendo de la libertad la ley que regula la interacción mutua (gegenseitiger Wechselwirkung). Aquí se asienta el tema del concepto de derecho, a saber, el de "una comunidad entre seres libres como tales" 52 . Por consiguiente, o la libertad se conjuga de manera intersubjetiva -esto es, distribuyéndose y delimitándose mutuamente-, o se autodestruye en la individualidad. De ahí que el 
objeto del derecho no sea lo que corresponde a cada uno, sino el ser libre en cuanto formando parte de una comunidad, en cuanto se realiza y constituye una comunidad con otros seres libres que, por ende, son iguales a él.

La influencia recíproca parte de una exhortación (Aufforderung) dirigida a la libre autodeterminación del sujeto, que decide actuar o no actuar, responder o abstenerse. En la medida en que el sujeto interpelado concibe y comprende la interpelación recibida, y en este sentido puede entender la finalidad, la exhortación misma denota una instancia compartida de racionalidad y de libertad, tanto respecto del emisor como del receptor. Sin esta influencia recíproca, la autoconciencia sería imposible ${ }^{53}$. El vínculo con el otro no se agota en el diálogo, sino que se eleva e involucra la práctica:

"el conocimiento de un individuo por otro está condicionado por el hecho de que el otro lo trate (behandle) como un ser libre [...]. Pero este modo de tratar (Behandlung) está condicionado por el modo de actuar del primero...".

Así, la reciprocidad se extiende a toda la comunidad. Prosigue Fichte:

"Por tanto, la relación de seres libres entre sí es la relación de una acción recíproca mediante la inteligencia y la libertad. Ninguno puede reconocer a otro si ambos no se reconocen mutuamente, y ninguno puede tratar al otro como un ser libre si ambos no se tratan así mutuamente" ${ }^{54}$.

En consecuencia, el derecho se erige sobre la relación entre seres libres y racionales, y no sobre las cosas $-\mathrm{o}$, a lo sumo, la cuestión surge cuando dos sujetos desean la misma $\operatorname{cosa}^{55}$-, en condiciones de igualdad y bajo el presupuesto de que en el proceso de realización de la libertad el sujeto distingue claramente de la otredad lo que es otrosujeto y otro-objeto. En efecto, una exhortación que apela a la comprensión racional supone un sujeto que ha limitado su libertad en relación con el otro, al cual también considera libre y racional ${ }^{56}$; pero ¿qué sucedería si la exhortación y el trato se orientaran hacia otro sujeto, no como si éste fuera tal, sino como si fuera un objeto? Desde ese momento no sólo se quiebran las condiciones del trato y se abre paso a la disputa física, sino que también se quiebran la libertad y la racionalidad de ambas partes. En la (pseudo-)relación opresor-oprimido el último tampoco es libre. Además, con la determinación del cuerpo propio, la cuestión queda absolutamente zanjada, y nadie confundirá sin intención a un hombre con un animal ${ }^{57}$.

21 Al enlazar la autoconciencia en una trabazón intersubjetiva, el concepto de comunidad de seres libres sobre el que se asienta el derecho gana factibilidad y espesor. Sin embargo, Fichte mismo aclara que hasta aquí sólo se trata de un plano meramente hipotético, en el sentido de que si se quiere formar una comunidad de seres libres, entonces tiene que valer la ley jurídica ${ }^{58}$. Y este tenor problemático se mantiene en la sección dedicada a la aplicación del concepto de derecho, específicamente respecto del derecho originario y del derecho de coacción. Al igual que en Schelling, la perspectiva desemboca en una situación aporética, situación que Fichte decide resolver de un modo significativo. El derecho originario -esto es, el derecho absoluto de la persona a ser causa primera en el mundo sensible- se manifiesta a través del cuerpo propio, que expresa la voluntad; y la actividad resultante, la transformación de los objetos según un concepto de fin (el trabajo), motiva que el sujeto se apodere de los objetos y los consigne como sus posesiones ${ }^{59}$. El problema emerge cuando el otro no reconoce o no respeta estas posesiones y, dado que no se ha delimitado lo legal y lo ilegal, ¿quién juzga? ¿Cada individuo? Así se desencadena una guerra infinita, un conflicto jurídico indecidible que culmina en la aniquilación o la expulsión ${ }^{60}$. 
22 Algo similar ocurre con el derecho de coacción, es decir, con la pretensión de que los demás se abstengan de usurpar o extender su influencia sobre la esfera de libertad del sujeto. Tal vez la seguridad recíproca pueda descansar en la creencia, buena fe, lealtad o confianza en que unos y otros se mantengan en el marco de los límites presupuestos. Sin embargo, estos lazos intersubjetivos que se sustentan en la moralidad se quiebran, junto con la seguridad recíproca, ni bien surge un conflicto, ni bien uno deja de confiar en las intenciones de otro. Por lo tanto, el derecho de coacción tampoco ofrece una cuidadosa separación entre lo legal y lo ilegal, porque la afirmación de la ley y la consiguiente interpretación del respeto o violación de la misma quedan relegadas a un plano meramente subjetivo ${ }^{61}$. La contienda se abre al infinito y, puesto que los rótulos agresor/agredido son intercambiables, se desencadena una situación escéptica en la que ambos tienen razón y ninguno tiene razón. Además, entre pares no hay justicia, porque no se puede ser al mismo tiempo juez y parte, y en tal caso sólo los más poderosos podrían ejecutar los castigos.

En consecuencia, ni el derecho originario ni el derecho de coacción se sostienen por sí mismos. La solución de tales aporías se logra con la institución de un poder (Macht) común, la República (gemeinen Wesen) o Estado que contiene en sí a las voluntades particulares junto con las limitaciones recíprocas, garantizando de este modo un espacio para la realización del sujeto en un marco de convivencia pacífica. El Estado, cuyo resorte último es la voluntad común, transforma el poder -hasta entonces físicoen derecho, y presenta el único suelo firme para el derecho natural -fuera del cual, y en sí mismo, se vuelve una ficción-, porque fuera del Estado no existe ninguna relación jurídica sustentable ${ }^{62}$. Según esta consideración, Fichte profundiza las aporías planteadas por Schelling, pero las canaliza en una institución que representa y apuntala la intersubjetividad, definida bajo los parámetros inseparables de la libertad y la igualdad.

\section{4.}

24 El periplo del presente trabajo, motivado por el intento de ahondar en las afinidades filosófico-jurídicas entre Schelling y Fichte, se inició con la cuestión hermenéutica que matiza la dicotomía entre continuidad y ruptura, no sólo porque comparten una declarada convalidación mutua, sino también porque se posicionan análogamente al momento de comprender la herencia crítica y proyectar el propio idealismo.

25 La comprensión filosófica permite advertir que la interpretación, así como no se mantiene en la linealidad, tampoco resulta arbitraria ni inventa un espíritu en otro para justificar la propia teoría; antes bien, extrae consecuencias, lo implícito de lo explícito, constitutivas de la coherencia del todo. Por ende, contraponer fragmentos de Schelling y de Fichte sin atender al sentido de conjunto es una batalla cuyo desenlace se encuentra definido de antemano. A su vez, reconstruir semejante totalidad -tarea nada fácil- conlleva el riesgo inmediato de soslayar las divergencias. Igualmente, que haya que comprender a Kant -incluso quizás mejor de lo que él se comprendió a sí mismolegitima una segunda revolución, un giro del giro copernicano donde el conocimiento objetivo pasa a un plano secundario frente a la primacía de la práctica. Este giro busca neutralizar simultáneamente el empirismo y el dogmatismo. En efecto, Fichte y Schelling convergen en el apuntalamiento del primer principio (el Yo, la libertad, la actividad autogenerada, sujeto-objeto, etc.), y en el caso del sujeto, el esfuerzo por 
realizar lo incondicionado y exteriorizar su voluntad (el pasaje de la determinabilidad a la determinación) se encuentra con la resistencia, aquello que lo inhibe u obstaculiza. Lo que Schelling llama "vida", la interacción con el mundo sensible, Fichte lo precisa y detalla como cuerpo propio, el ámbito desde el cual se articula la relación recíproca. En este punto los senderos se bifurcan.

26 Schelling, por una parte, aunque distinga la limitación física de la moral, en la relación con el otro exige una voluntad auto-afirmativa, exige que el otro explicite claramente que no es un objeto más de la naturaleza. Pero la concordancia ética no redunda en una coincidencia fáctica, de lo cual se sigue el requerimiento de una regulación jurídica, que tampoco resuelve el problema si no se instituye un poder que mantenga a raya las voluntades en pugna. La potenciación inicial del conflicto -inherente a la propia afirmación de la voluntad- cierra todos los caminos y torna estéril al derecho natural mismo. La individualidad no organizada en una instancia común genera un estado de naturaleza autodestructivo. Fichte, por otra parte, al situar al otro en la configuración del sí-mismo, genera precondiciones igualitarias para el reconocimiento y para una distribución social de la libertad, de modo tal que la realización del sujeto corre en paralelo y en interacción con la realización de los demás, porque nadie puede adjudicarse una esfera de acciones posibles sin convalidar ese mismo acto en todos los otros. Lejos del atomismo autosuficiente, el individuo se halla atravesado cultural, lingüística y educativamente, por la comunidad de pertenencia. Además, Fichte despeja la posibilidad de subsumir al otro al plano fenoménico, porque el cuerpo propio no se reduce a una simple connotación empírica, sino que implica la manifestación sensible de la libertad, aunque permanezca pasivo o no responda. Previo al conflicto hay un cierto entendimiento o racionalidad compartida que se plasma en el diálogo y en el trato, y que posibilita una relación jurídica.

27 Si esta reconstrucción se hubiera detenido aquí, las divergencias entre Schelling y Fichte parecerían insoslayables. Sin embargo, comulgan en los puntos nodales. Fichte no desestima el conflicto, pues ni bien lo introduce echa por tierra la comunidad incipiente y la reciprocidad; lo que sí descarta -en términos de Schelling- es la autoafirmación de la voluntad individual en cuanto instancia vertebradora que conlleva una infructuosa universalidad ética y que, en lo fáctico, precipita la dominación. Pero Fichte no ensaya una concordancia sustentada en la buena fe, ni busca la conciliación dialéctica de voluntades contradictorias, ni pretende construir lo común a partir de individualidades que se autoafirman; es decir, no insiste en resolver la conflictividad en una dimensión a-estatal. En la medida en que el problema reside en concebir la voluntad individual como eje jurídico-político escindido e independiente de la racionalidad compartida o del reconocimiento mutuo, el desafío consiste en anteponer lo universal a lo particular sin que, tal como lo prevé Schelling, lo primero anule lo segundo. En el estado de naturaleza la universalidad siempre se encuentra plasmada de modo problemático. Al rechazar la articulación del concepto de derecho basado en la voluntad individual auto-afirmativa, y al focalizarlo en la voluntad común de seres libres -en rigor, única instancia a partir de la cual cada individuo puede ser libre-, Fichte refuerza el concepto que le permitirá superar las aporías del derecho originario y del derecho de coacción, la República o Estado, concepto que termina de afirmar la comunidad incipiente del reconocimiento y la reciprocidad. Aunque en la Nueva Deducción Schelling explícitamente no dé este último paso, presenta las aporías que desembocan en la autodisolución del derecho natural, cercena las posibilidades de la libertad atómica en el estado de naturaleza, y prepara el terreno para que las figuras 
del derecho originario y del derecho de coacción resulten inescindibles de un poder físico-jurídico, aspectos con los cuales comulga Fichte y de los cuales se sirve tanto para despejar la representación de una comunidad armónica no cristalizada en instituciones, como para subsumir las libertades y limitaciones de los individuos a la órbita del Estado.

\section{NOTAS}

1. Además de las abreviaturas convencionales, utilizaremos ND para Neue Deduktion des Naturrechts. Por otra parte, quiero agradecer el estímulo y los valiosísimos aportes de Emiliano Acosta, Ana Carrasco Conde y Edgard Maraguat.

2. FICHTE, ZE, GA I/4, $220 \mathrm{n}$.

3. FICHTE, ZE, GA I/4, $234 \mathrm{n}$.

4. FICHTE, ZE, GA I/4, 238-239.

5. FICHTE, ZE, GA I/4, $265 \mathrm{n}$.

6. FICHTE, ZE, GA I/4, $267 \mathrm{n}$.

7. SCHELLING, Abhandlungen zur Erläuterung des Idealismus der Wissenschaftslehre, SSW I/1, 409-413 (trad. V. Serrano, Panorama general de la literatura filosófica más reciente, Madrid, Abada, 2006: 122-126). En el curso del tratado IV (SSW I/1, 403 ss.; trad. 115 ss.) Schelling critica la visión prosaica del idealismo unilateral, en cuanto excluye al realismo, y propone una síntesis que integra ambas posiciones. Cuando el kantismo presenta una cosa en sí incognoscible, se precipita en un realismo ingenuo cuyo problema ulterior reside en hacer concordante la representación y el objeto referido, y se genera el dualismo entre lo suprasensible y lo sensible. De ello se desprende que Schelling considere que la filosofía teórica de Kant quedó incompleta o indeterminada, y afirma: "Kant tiene que simbolizar este fundamento suprasensible en la filosofía teórica, y entonces dice que las cosas en sí como tales dan la materia a nuestras representaciones. Fichte puede carecer de esta presentación simbólica, porque él no ha tratado la filosofía teórica, como Kant, separada de la práctica. Precisamente en esto consiste el mérito más propio del último: en que el principio que Kant sitúa en la cima de la filosofía práctica (la autonomía de la voluntad), lo extiende a principio de toda la filosofía, y por eso deviene fundador de una filosofía que, con derecho, se puede llamar filosofía superior, porque en su espíritu ella no es ni sólo teórica ni sólo práctica, sino ambas a la vez" (SW I/1, 409, trad. 122). Poco más abajo (SW I/1, 413; trad. 126-127) Schelling insiste en defender a la Doctrina de la Ciencia de la acusación de idealismo ingenuo, y reinterpreta le mérito de Fichte como "identidad originaria de la filosofía teórica y práctica en nosotros $"(413 ; 127)$.

8. SCHELLING, System des transscentalen Idealismus, SW I/3, 330-331; trad. cast. V. López Domínguez y J. Rivera de Rosales, Barcelona, Anthropos, 1988: 139.

9. FICHTE, GWL, GA I/2, 252. "A los futuros críticos de este escrito les pido que ingresen desde el todo y observen cada pensamiento particular desde la perspectiva del todo", 253. scHeLLING, Vom Ich als Princip der Philosophie, SW I/1, 151: “[hago] aquí solamente una única petición a los lectores y críticos de este escrito: o bien no lo lean en absoluto, o bien lo lean en su conexión total (in ihrem ganzen Zusammenhang); [es decir,] o bien se contengan de todo juicio, o bien juzgue al autor según el todo, y no según posiciones particulares separadas del conjunto (Zusammenhang)". Trad. cast. Pérez-Borbujo y Giner Comín, Madrid, Trotta, 2004: 59. Al tema del espíritu y la letra Fichte 
le dedica, además de varios pasajes, los opúsculos ("Sobre el espíritu y la letra en la filosofía": 103-133, "Sobre la diferencia entre el espíritu y la letra en la filosofía": 135-167, "Quiero investigar en qué se diferencia en general el espíritu de la letra en la filosofía": 207-220) compilados en Filosofía y estética. La polémica con Schiller, introd., trad. y notas F. Oncina Coves y M. Ramos, Valencia, Universidad de Valencia, 1998.

10. FICHTE, DS, edición bilingüe y trad. M. Ramos y F. Oncina Coves, Madrid, Istmo, 2002: 128-129 (GA I/3, 61).

11. FICHTE, EE, GA I/4, 184.

12. FICHTE, ZE, GA I/4, 221 (subrayado nuestro).

13. FICHTE, ZE, GA I/4, 222. Su juicio definitivo y negativo lo hará público Kant en 1799: “... declaro que considero la Wissenschaftslehre de Fichte como un sistema completamente insostenible", en F. ONCINA COVES y M. RAMOS, "Introducción” a FICHTE, Filosofía y estética, 36-39, la cita: 36 (Ak.-Ausg. XII, 370).

14. FICHTE, ZE, GA I/4, 231-232 n; la cita en 232 .

15. SCHELLING, Vom Ich, SSW I/1, 152-153; trad. 60-61.

16. SCHELLING, Abhandlungen, SSW I/1, 375; trad. 81.

17. SCHELLING, Vom Ich, SSW I/1, 157; trad. 66.

18. SCHELLING, ND $\S 1$, SSW I/1, 247; trad. F. Oncina Coves, con introducción y notas, en Thémata. Revista de Filosofía, n 11, 1993: 226-227.

19. SCHELLING, ND $\S 2$, SSW I/1, 247; trad. 227. En su brillante exposición E. AcosTA presenta la deducción del derecho en Fichte y Schelling a partir de la exigencia absoluta de realización de la libertad absoluta, el ideal al que se orienta el esfuerzo: " $\mathrm{Zu}$ einem alternativen Begriff von Pluralismus anhand Schellings und Fichtes Deduktionen des Naturrechts", en Congreso Internacional Fichte-Gesellschaft, Bruselas (2009), disponible en: www.europhilosophie.

eu (URL: http://www.europhilosophie.eu/recherche/IMG/pdf/16a-Acosta-Fichte-SchellingRechtsdeduktionen.

pdf).

20. Véase, por ejemplo, la polémica de Fichte con el dogmatismo en EE, GA I/4, 188 ss. "La disputa entre el idealista y el dogmático es propiamente la de si la independencia (Selbständigkeit) del Yo [= autonomía] debe ser sacrificada frente a la independencia (Selbständigkeit) de la cosa, o al revés, la de la cosa ante la del Yo", 193.

21. SCHELLING, Vom Ich, § 4; SSW I/1, 170-172, trad. 78-79.

22. FICHTE, GWL $\S 1, G A I / 2,259$. "Aquello cuyo ser (esencia) sólo consiste en ponerse a sí mismo como siendo es el Yo como sujeto absoluto" y concluye más abajo: "El Yo pone originaria y absolutamente su propio ser", 260. Recordemos que Fichte deduce esta "acción del espíritu humano" o "carácter puro de la actividad en sí" (258-259) de la proposición "Yo soy", proposición que Schelling analiza en Vom Ich § 3, y en ND §§ 55 y 60. Sobre el ser en Fichte: J. L. VILLACAÑAS, "Ser y existir: la estrategia de Fichte contra el Nihilismo”, en Daimon. Revista de filosofía, Número 9, 1994: 135-154.

23. FICHTE, EE, GA I/4, 186; ZE, GA I/4, 215, 216-217, 245; VND, GA I/4, 271.

24. SCHELLING, ND § 3, SSW I/1, 247; trad. 227.

25. SCHELLING, ND § 4, SSW I/1, 248; trad. 227.

26. SCHELLING, ND § 5, SSW I/1, 248; trad. 227. Afirma en otro texto: "Mi destino (Bestimmung) en el criticismo es, en efecto, el esfuerzo (Streben) hacia una mismidad (Selbstheit) invariable, una libertad incondicionada, una actividad ilimitada", y agrega en el párrafo siguiente: “¡Sé!, es la exigencia suprema del criticismo"; schelling, Philosophische Briefe über Dogmatismus und Kriticismus, IX, SSW I/1, 335 (trad. V. Careaga, Madrid, Tecnos, 1993: 94). Véase también: SSW I/1, 308. El paralelismo entre ser y saber se encuentra desde el comienzo del § 1 de Vom Ich: "Quien quiere saber algo, quiere a su vez que su saber tenga realidad". Y sobre el final del § 1: "El fundamento último de toda realidad es, en efecto, algo pensable sólo por sí mismo, es decir, pensable por su ser, que sólo 
en la medida en que está pensado es, dicho brevemente, en cuanto el principio del ser y el principio del pensar coinciden". SSW I/1, 162-163 (trad. 71-72); también § 3.

27. SCHELLING, ND § 7, SSW I/1, 248; trad. 227.

28. SCHELLING, ND $\S 132$, SSW I/1, 273; trad. 245: "En efecto, la autonomía debe regir absolutamente sobre la heteronomía. Todo lo que es objeto debe comportarse de manera absolutamente pasiva frente a la autoactividad de un sujeto moral".

29. SCHELLING, ND § 8, SSW I/1, 248-249; trad. 227-228.

30. SCHELLING, ND §§ 9-12, SSW I/1, 249; trad. 228.

31. Streben en FICHTE: GWL $§ 5$, GA I/2, 385 ss.; en 397 afirma: "la actividad pura del Yo, la actividad que regresa en sí misma, es, en relación con un objeto posible, un esfuerzo; y además, según la anterior demostración, un esfuerzo infinito. Este esfuerzo infinito, en su extensión (hinaus) infinita, es la condición de posibilidad de todo objeto; si no hay esfuerzo, no hay objeto". El esfuerzo reúne la actividad objetiva, dirigida hacia un objeto, con los fines supremos, con los Ideales (402 ss.). Sobre la articulación entre lo determinable y lo determinado, Fichte se ocupa en WLnm-H (GA IV/2; véase, por ejemplo, §§ 2-5, 9), así como de libertad y limitación (§§ 6-7). Observemos algunos pasajes: "La libertad, por tanto, es el fundamento supremo, y la condición primera de todo SER y de toda conciencia" ( $§ 3, \mathrm{GA}$ IV/2, 47); “[el] yo como actividad real es, en efecto, un pasaje ( Übergehen) de la DETERMINABILIDAD a la DETERMINACIÓN -esto es, la capacidad práctica o la libertad ABSOLUTA elige entre [o extrae de] la esfera de lo determinable una parte, lo determinado" (\$ 4, 50). "La acción es la actividad resistida [u obstaculizada] continuamente, y sólo mediante esta SíNTESIS con la resistencia llega a ser una actividad intuible" $(\S 5,56-57)$.

32. FICHTE, GNR “Einleitung", GA I/3, 313; trad. Villacañas, Oncina Coves y M. Ramos (= FDN), Madrid, Centro de Estudios Constitucionales, 1994: 103.

33. FICHTE, GNR "Einleitung”, GA I/3, 314-316; FDN, 104-105.

34. FICHTE, GNR § 1, GA I/3, 331; FDN, 118.

35. FICHTE, GNR § 1 Cor., GA I/3, 332; FDN, 119. El § 2, que se erige como consecuencia del $\S 1$ (primer teorema), reza así: "El ser racional, mediante este poner su facultad para la actividad causal libre, pone y determina un mundo sensible exterior a él”, GNR 335; FDN 121. El mundo sensible se deduce como actividad reprimida del Yo y, por ende, como su producto.

36. FICHTE, GNR § 5, GA I/3, 363; FDN, 149. La temática se extiende al § 6 y Fichte la retoma en $§ 11$. En Abhandlungen, SW I/1, 388-390 (trad. 99-100), Schelling deduce lo vivo como lo análogo y visible de lo espiritual, y el cuerpo como huella (Abdruck) del alma.

37. FICHTE, GNR § 7, GA I/3, 384; FDN, 171.

38. En el artículo citado, E. AcosTA distingue el reconocimiento por contradicción o disenso, mediante el conflicto (Schelling), del reconocimiento por unidad o consenso, mediante el respeto de la esfera de cada individuo, la colaboración y la educación (Fichte). Tomaremos esta perspectiva como premisa.

39. En la introducción a la citada trad. de SCHELLING, ND, F. ONCINA COVES señala la precariedad del contrato según la nota al § 85, SW I/1, 263 (trad. 238). También F. ONCINA COVES, "El iusnaturalismo agónico: la teoría fichteana del contrato", en V. LóPEZ DOMínGUEZ (ed.), Fichte 200 años después, Madrid, Complutense-UNED, 1996: 75-97. De la nota al § 85 de ND se desprende que Schelling reclama un fundamento para asegurar cada contrato, lo cual llevaría a una "serie infinita de contratos" (SW I/1, 263 n; trad. 238 n) en el contexto de los derechos originarios y de la voluntad individual. Aunque Fichte reivindique el contrato de ciudadanía, que implica unidad social, protección, etc. (GNR $\S 17$, GA I/4, 15-18; FDN 268-271), no lo realiza como precondición del Estado, ni como secuencia inherente al estado de naturaleza, sino al revés, en cuanto deberes y derechos de los ciudadanos en sus relaciones recíprocas y en cuanto naturaleza de la cosa pública. Así, los contratos no se consuman previamente a la conformación del Estado, sino a 
partir de éste. Por ende, Schelling y Fichte coinciden en señalar y profundizar la problematicidad de los acuerdos y de los derechos, como veremos más abajo, en una eventual situación a-estatal.

40. SCHELLING, ND §§ 11-13, SSW I/1, 249; trad. 228.

41. SCHELLING, ND § $15 \mathrm{n}$, SSW I/1, $250 \mathrm{n}$; trad. $229 \mathrm{n}$.

42. SCHELLING, ND § 21, SSW I/1, 251; trad. 229. La perspectiva continúa en §§ 26-27.

43. SCHELLING, ND § 45, SSW I/1, 255; trad. 232.

44. SCHELLING, ND §§ 46-47, SSW I/1, 255; trad. 232-233.

45. SCHELLING, ND §§ 53, SSW I/1, 257; trad. 233.

46. SCHELLING, ND §§ 65, 67-68, SSW I/1, 259-260 (trad. 235-236); ND § 140, SSW I/1, 275 (trad. 246-247). Como bien señala F. ONCINA coves (nota a la trad., 253), estos tres principios guardan familiaridad con los de KANT en "Acerca del refrán: «Lo que es cierto en la teoría, para nada sirve en la práctica»" (Ak.-Ausg. VIII, 290).

47. V. gr. SCHELLING, ND § 101 (SSW I/1, 267; trad. 241), §§ 113-115 ss. (SSW I/1, 269-270 ss.; trad. 242-243 ss.), § 139 (SSW I/1, 275; trad. 246).

48. SCHELLING, ND $\S ~ 156,158,160$ (SSW I/1, 278; trad. 249), respectivamente.

49. SCHELLING, ND § 161, SSW I/1, 279; trad. 249.

50. SCHELLING, ND § 162, SSW I/1, 279; trad. 249. La "nueva ciencia del derecho" (§ 163; SSW I/1, 279; trad. 249-250), precisamente aquella que no se deduzca de la moral y que no se empantane aporéticamente en la lucha natural, tendrá que resolver la configuración de la fuerza física en cuanto fuerza jurídica; es decir, tendrá que identificar el poder con la ley. Aunque Schelling no lo explicite aquí, la resolución sólo se puede dar en el interior de un Estado, al que posteriormente define como "organismo objetivo de la libertad". schelLing, Vorlesungen über die Methode des akademischen Studiums, SSW I/5, 382; véase también: 232, 260, 315-316.

51. FICHTE, GNR “Einleitung”, GA I/3, 319; FDN, 108.

52. FICHTE, GNR “Einleitung”, GA I/3, 320; FDN, 109.

53. FICHTE, GNR § 3, GA I/3, 342 ss.; FDN, 128 ss.

54. FICHTE, GNR § 4, GA I/3, 351; FDN, 137. No hay que comprender esta bicondicionalidad desde un punto de vista temporal, preguntando por ejemplo quién reconocerá primero al otro, porque entonces conduciría al infinito y la serie jamás se podría iniciar. La práctica ya ha resuelto este problema teórico: o en el trato mismo los sujetos se presuponen libres, o el trato se destruye a sí mismo. Pero sucede que, como veremos más abajo, el trato y el reconocimiento se mantienen en una situación hipotético-problemática en la medida en que no se establezca una institución (el Estado) que fundamente la presuposición de libertad en el vínculo recíproco.

55. FICHTE, GNR § 4, GA I/3, 360; FDN, 146.

56. FICHTE, GNR § 3, GA I/3, 345-347; FDN, 131-133.

57. FICHTE, GNR § 4, GA I/3, 352-353; FDN, 138-139; § 6 Cor., GA I/3, 380 ss.; FDN, 166 ss. Sobre opresor y oprimido: FICHTE, "Sobre el espíritu y la letra en la filosofía”, en Filosofía y estética, 120.

58. FICHTE, GNR § 7, GA I/3, 387; FDN, 174.

59. FICHTE, GNR §§ 10-11, GA I/3, 404-407; FDN, 192-195. Si bien aquí Fichte alude a la propiedad (Eigenthum), se sobreentiende que mientras no haya un Estado que garantice el reconocimiento recíproco será, en rigor, una mera posesión (Besitz): GNR § 12, GA I/3, 417-418; FDN, 206-207.

60. FICHTE, GNR § 12, GA I/3, 415; FDN, 203.

61. FICHTE, GNR § 13, GA I/3, 424; FDN, 214.

62. FICHTE, GNR § 15, GA I/3, 430-432; FDN, 220-222. El carácter ficcional del estado de naturaleza, o del derecho originario en una instancia a-estatal (de este tema nos hemos ocupado en "El estado natural del hombre es el Estado", en E. AcosTA (ed), Estudios sobre Fichte, en www.europhilosophie.eu, 2010: 33-68; URL: http://www.europhilosophie-editions.eu/fr/ spip.php?article36), Fichte lo advierte desde el comienzo, GNR § 9, GA I/3, 403; FDN, 192. Luego, el 
sentido de este tratamiento se comprende en relación con la legislación civil y la legislación penal (GNR § 16, GA I/3, 434-435; FDN, 225), desarrolladas en la segunda parte (§§ 18-20).

\section{RESÚMENES}

This article analyze the relationship between Schelling and Fichte in some philosophical and legal questions, previously developing mutual references and the problem of understanding in the philosophy (point 1). In Neue Deduktion des Naturrechts Schelling exposes several concepts that, in his own philosophy project, agrees with Fichte's Wissenschaftslehre in fundamental points, particularly with Grundlage des Naturrechts (point 2). On basic coincidence, the most visible is the intersubjectivity and the conflict that appears with the other, which leads to the natural right problem. Schelling and Fichte share the skepticisms about original right and coaction right, but Fichte neutralizes war (that involves) all against all with State's institution (point 3). Although, they seem to find different results, they agree in diagnostic about the individual will and the necessity to establish a common instance.

\section{ÍNDICE}

Keywords: philosophical understanding, first principle, freedom and necessity, intersubjectivity, conflict, natural right

\section{AUTOR \\ MARIANO GAUDIO}

Universidad de Buenos Aires 